\title{
Exploitation des usines marémotrices compte tenu des limitations imposées aux vitesses de variation des débits
}

\author{
The operation of tidal power stations \\ in view of the limitations imposed \\ on the speed of discharge variations
}

\author{
PAR R. GIBRAT \\ INGÉNIEUR-CONSEIL, \\ PROFESSEUR a L'ÉCOLE SUPÉRIEURE DES MINES DE PAllis, \\ PRÉSIDENT DU COMTSÉ TECHNIQUE DE IAA SOGIETTE HYDROTECHNIQUE DE FHANCE
}

\begin{abstract}
L'utilisation simultanée du site d'une usine marémolrice à d'antres fins que la production d'énergie impose aux vilesses de variation des débits des limiles qui, pour la Rance, sont fixées par l'Administration $\dot{a} \pm 3 \mathrm{~m}^{\mathrm{s}} / \mathrm{s}^{2}$. Une solution commode a cette linitation consiste a mettre en route (ou à arrêter) chaque anité de l'usine à la suite l'une de l'autre.

Au démarrage et d̀ l'arrêt en simple effet, la condition de rendement maximum valable, au départ, d'après les études antérieures, fait que la différence d'énergie des deux fonctionnements, brutal ou graduel, de chaque groupe, est du mêne ordre (inférieure $\dot{a} 3 \%$ pour la Rance). D'autre part, la perte de chute subie par la dernière unité démarrée et la première arrêtée est négligeable.
\end{abstract}

En double effet, au remplissage, la question met en jeu le vannage des turbines et celui des bannes proprement dites. Tris complexe en theorie, elle peut être résolue, pour la Rance, par des calculs namériques dans quatre hypotheses distinctes, purtant tontes du fonctionnement des groupes d puissance maximum. La comparaison des résultats énergétiques révèle un net avantage de deux de ces solutions: lune. consistant $\dot{a}$ fuire fonctionner toutes les turbines a puissance maximum au départ, puis à en découpler dix à cerfaine phase du remplissage; l'autre consistant à faire fonctionner tontes les turbines couplées, tout le temps, mais à un régime qui intègre l'effet du récouplage partiel précédent.

Mais ce net avantage et la faible différence des deux soiutions dégagés montre tont $\dot{a}$ la fois l'intérêt et les limitations des rechcrches théoriques à faire.
The use of a tidal power station site at the same time for producing power and for other purposes imposes limits on the speed of the discharge variations, which for the Rance have been fixed by the Administration at $\pm 3 \mathrm{~m}^{9} / \mathrm{s}^{2}$. A convenient solution of this limitation consists in starting up (or stopping) each power station unit one after the other.

In starting up or shutting down with one wall flow it is seen that by having the condition of maximum available efficiency (as determined from previous studies) at the beginning of flow, there is little difference between the power produced by the two methods of operation of each unit, sudden and gradual (the difference for the Rance is less than three in a thonsand). On the other hand the head loss in the last unit started up, and the first unit shut down is negligable.

With the flow active in both directions the problem involues the gate mandures for the turbines, and the gates themseloes. Ver!y complicated in theory this can be solved for the Rance by numerical calculations brised on fous distinct hypotheses, all beginning from the operation of the units at maximum power. Comparison of the power results reveals a clear advantage for two solutions : one in which all the turbines work at maximum power at the beginning and then ten are cut off at a certain stage of the filling; in the other all the turbines work all the time but according to a puttern which integrates the effect of the preceding partial shut down.

But this clear adnantage and the small difference between the two solutions derived shows at the same time the interest and the limitations of the theoretical research to be done. 
L'existence d'un port en amont ou en aval d'une usine marémotrice, les exigences de la navigation à travers le barrage, fixent, par nature, des limites anx vitesses de variation des débits. Ainsi, lors de l'enquêtte sur l'usine de la Rance, il est apparu nécessaire de délimiter les variations rapides du débit. D'une manière précise, les pouvoirs publics nous demandent qu'une variation de débit égale à la moitié du débit maximum turbinable prévu alors, soit $2.750 \mathrm{~m}^{3} / \mathrm{s}$, ne s'effectue pas en moins d'un quart d'heure, cette condition pouvant toutefois être modifiée dans un sens ou dans l'autre d'après les renseignements recueillis lors de la mise en fonctionnement progressive de l'usine.

Il s'agit donc, en chiffres ronds, d'une variation totale limite de $3 \mathrm{~m}^{3} / \mathrm{s}^{2}\left(2.700 \mathrm{~m}^{3} / \mathrm{s}\right.$ par quart d'heure). Nous rappelons que le débit de la marée lors des plus grandes vives eaux peut atteindre près de $20.000 \mathrm{~m} \mathrm{~m}^{\mathrm{n}} / \mathrm{s}$. On pourrait estimer que cette limite, du côté de l'estuaire par exemple, devrait varier avec le niveau de l'eau plus fort à pleine mer qu'à basse mer (*). Mais les formules donnant le débit total traversant le barrage $Q(z, t)$ ou $Q(h, t)$ équivalent évidemment à des lois $Q(t)$. Ainsi dans le premier cas nous avons la relation $-\mathrm{S}(d z / d t)=\mathrm{Q}$ traduisant les variations du volume d'eau retenu et définissant $z$ en fonction du temps (**). En effet, supposons par exemple que nous ayons la consigne $|d Q / d t| \leqslant f(z, t)$; les lois limites :

$$
d \mathrm{Q} / d t= \pm f(z, t)
$$

équivalent aux équations :

$$
d / d t[-\mathrm{S}(d z / d t)]= \pm f(z, t),
$$

équations différentielles du second ordre qui, pour des conditions initiales définies en $z$ et $Q$, permettent de déterminer $z(t)$ et donc $Q(t)$.

Le cas de $|d \mathrm{Q} / d t| \leqslant g(h, t)$ avec $h=h(t)$ est évident.

On aurait un résultat de même forme si on voulait limiter l'accélération de l'eau, soit $d / d t[(\mathrm{Q} / s(z)], s(z)$ étant la section mouillée; nous aurions alors les lois limites :

$$
\mathrm{Q}=s(z)(\alpha \pm \beta t)
$$

équivalentes aux équations :

$$
-\mathrm{S}(z)(d z / d t)=s(z)(\alpha \pm \beta t)
$$

équations du premier ordre définissant $z$ en fonction du temps et donnant donc encore des lois limites de la forme $Q(t)$.

Les études suivantes utiliseront done une loi $Q(t)$ fixée à l'avance, mais pour la compréhension du phénomène et les premières applications, il nous parait suffisant d'admettre la variation limite demandée à l'enquête de $\pm 3 \mathrm{~m}^{3} / \mathrm{s}^{2}$. Deux solutions sont à priori possibles : une variation continue identique pour toutes les unités de l'usine, ou au contraire une mise en route (ou un arrêt) de chaque unité l'une à la suite de l'autre. La deuxième solution seule demande une étude, c'est d'ailleurs la seule commode.

\section{I. - DÉMARRAGE DE L'USINE}

Le démarrage instantané, ou en quelques minutes, des 38 groupes actuellement prévus pour la Rance, parfaitement possible si on se borne aux machines, ne permet pas de respecter la condition limite. Le rendement étant maximum

(*) Nos notations restent celles de notre article sur « l'Energie des Marées », publié dans le Bulletin de la Sociélé Française des Electriciens, en mai 1953 (n ${ }^{\circ} 29$ ) et de notre précédente communication à la Société Hydrotechnique de France publiée dans la Houille Blanche, « Premiers critères de comparaison des diverses turbomachines au point de vue des usines marémotrices $\gg$, en janvier $1954\left(n^{\circ} 1\right)$ :

$h$ niveau d'eau en aval du barrage, $z$ en amont.

$l$ est le temps,

S (z) définit la surface du plan d'eau de l'estuaire pour le niveau $z$.

$\left(^{*}\right)$ Ceci suppose que les lois $Q(t)$ sont a variation assez lente pour qu'au moins à une certains distance du barrage le mouvement des eaux puisse être considéré comme une suite de régimes permanents, ce qui paraît démontré par nos essais en modèle réduit. $[(\partial \eta / \partial q)=0]\left(^{\star * *}\right)$, nous avons un débit par groupe de $100 \mathrm{~m}^{3} / \mathrm{s}$ par exemple, il faut donc démarrer un groupe environ toutes les demi-minutes et compter un peu plus de vingt minntes pour mettre tous les groupes en route. Par contre, un à-coup, même pratiquement instantané, de $100 \mathrm{~m}^{3} / \mathrm{s}$, est à l'intérieur d'un intervalle de quelques dizaines de secondes certainement supportable car il met en jeu des volumes d'eau négligeables. Nous pouvons donc raisonner comme si le nombre de groupes variait continûment. Si à un instant, pour une hauteur de

$\left({ }^{\star \star *}\right)$ On se rappelle que cette condition $[(\partial \eta / \partial q)=0]$ est valable pour tous les fonctionnements dont la cote de départ (pour le turbinage), ou d'arrivée (pour le nompage) est donnée. Nous montrerons dans des publications ultérieures qu'elle doit par contre être modifiée si le prix de l'énergie varie non seulement avec le temps, mais avec la puissance fournie, ce qui peut être le cas des équipements marémoteurs de grande importance vis-àvis des réseaux qu'ils alimentent (Chausey, par exemple). 
chute $H$, le nombre de groupes en marche est $n$, nous savons par nos études antérieures que chaque groupe doit avoir le même débit inconnu $q(\mathrm{H})$, d'où la première équation :

$$
n q=\mathrm{Q}(t)
$$

Il s'agit de rendre maximum l'énergie produite :

$$
\mathrm{E}=\int n \cdot \mathrm{N}^{\prime}(q, \mathrm{H}) d t
$$

Ceci s'obtiendra en rendant maximum, pour chaque valeur de $t$ (ou de $\mathrm{H}$ ), $n \mathrm{~N}$ ou $\mathrm{N} / q$, ou encore le rendement $\eta$ puisque $\mathrm{N}=\eta q \mathrm{H} . n \mathrm{~N}$, ou $(\mathrm{N} / q) \mathrm{Q}(t)$, ou $\eta \mathrm{H} \mathrm{Q}(t)$ est en effet maximum, $\mathrm{H}$ et $t$ étant donnés, pour :

$$
\frac{\partial \eta}{\partial q}=0
$$

Par suite, tout le long du démarrage pendant les vingt minutes correspondantes, chaque groupe fonctionne au débit donnant le rendement maximum pour la hauteur de chute correspondante. Ce débit changeant peu, l'équation (1) montre que $n$ varie à peu près comme $Q(t)$, c'està-dire linéairement. Mais la détermination de la loi exacte de mise en route des groupes ne pose aucune difficulté. Le point représentatif $(q, \mathrm{H})$ de la colline individuelle du premier groupe mis en route va de $a$ en $b$ sur $\partial \eta / \partial q=0$, puis quitte cette courbe suivant l'extrémale $b m$ dès
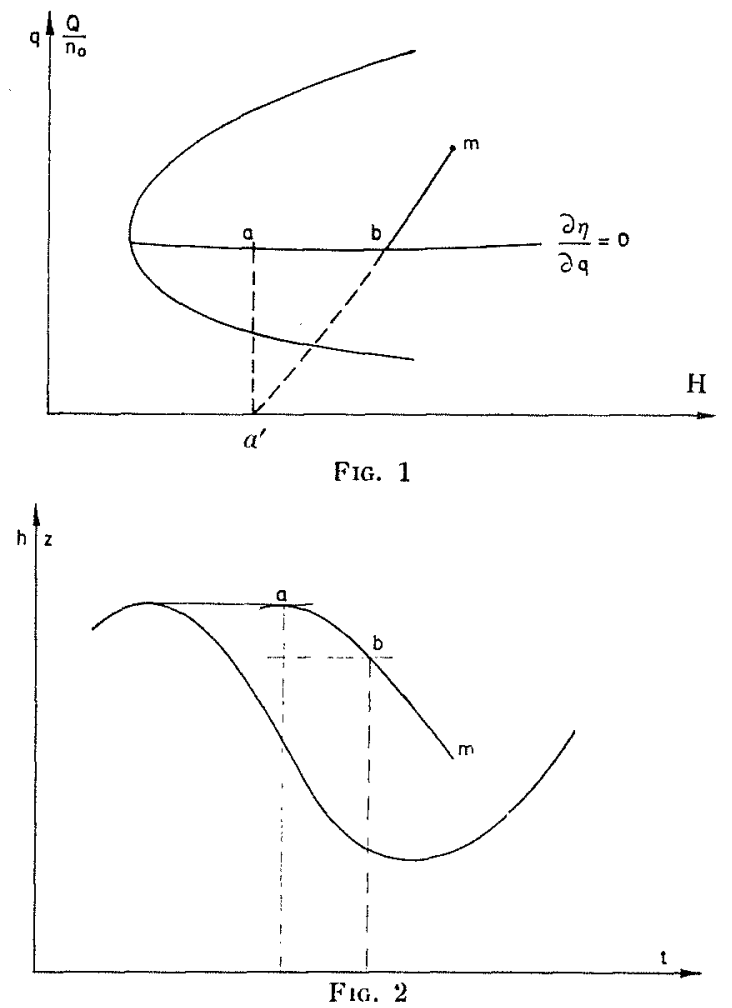

que $n$ a atteint sa valeur maximum $n_{0}$ (38 par exemple). Le point représentatif $\left[\left(\mathrm{Q} / n_{0}\right), \mathrm{H}\right]$ de la colline totale de l'usine se déplace par contre de $a^{\prime}$ en $b$, suivant la loi définie par la loi $Q(t)$, l'extrémale $b m$ étant commune aux deux collines.

Notre fonctionnement classique de l'usine totale en vidage par exemple commence ainsi sans aucune modification au point $b$ à une hauteur $z_{b}$ différente de la hauteur de début de fonctionnement $z_{a}$. En a la tangente à notre extrémale nouvelle, compte tenu de la limitation $Q(t)$, est horizontale.

Dans le cas où il y aurait plusieurs types de groupes dans l'usine avec des collines difrérentes, il faudrait évidemment à chaque instant mettre en service le type de groupe qui a le plus grand rendement maximum pour la chute à l'instant considéré. Les équations suivantes le démontrent sans difficulté. On aura par exemple pour deux types de groupes:

$$
\begin{gathered}
n_{1} q_{1}+n_{2} q_{2}=\mathrm{Q}(t) \\
\mathrm{E}=\int\left(n_{1} \mathrm{~N}_{1}+n_{2} \mathrm{~N}_{2}\right) d t
\end{gathered}
$$

l'extremum de $d \mathrm{E} / d t$ pour $\mathrm{Q}(t)$ donné correspond aux conditions :

$$
\frac{\partial \eta_{1}}{\partial q_{1}}=0, \frac{\partial r_{2}}{\partial q_{2}}=0
$$

puis :

$$
\begin{array}{r}
\delta\left(\frac{d \mathrm{E}}{d t}\right)=\mathrm{N}_{i} \delta n_{1}+\mathrm{N}_{2} \delta n_{2}=\delta n_{\mathrm{t}}\left(\mathrm{N}_{1}-\mathrm{N}_{2} \frac{q_{1}}{q_{2}}\right) \\
=q_{1} \mathrm{H} \delta n_{1}\left[\eta_{1}\left(q_{1}, \mathrm{H}\right)-\eta_{2}\left(q_{2}, \mathrm{H}\right)\right]
\end{array}
$$

Si, au début du démarrage, on a :

$$
\eta_{1}\left(q_{1}, \mathrm{H}\right)>\eta_{2}\left(q_{2}, \mathrm{H}\right)
$$

on commencera par mettre en roule, l'un apris l'autre, les groupes du type 1. Mais il se peut que, avant que l'on ait mis en route la totalité des groupes du type 1 , le classement des rendements maxima $\eta_{1}\left(q_{1}, \mathrm{H}\right), r_{2}\left(q_{2}, \mathrm{H}\right)$ (qui suivent des lois de variation différentes en fonction de la hauteur de chute $H$ ) vienne à s'inverser; dans ces conditions, dès que l'on aura :

$$
\eta_{1}\left(q_{1}, \mathrm{H}\right)<\eta_{2}\left(q_{2}, \mathrm{H}\right),
$$

on cessera la mise en route des groupes du type 1 au profit des groupes du type 2 , et on ne reprendra la mise en route des groupes du type 1 qu'après achèvement de la mise en route des groupes du type 2 ou encore (si le cas se produit avant cet achèvement) lors d'une nouvelle inversion du classement des rendements maxima $n_{1}\left(q_{1}, \mathrm{H}\right), n_{2}\left(q_{2}, \mathrm{H}\right)$. 
Pour en revenir au cas simple d'un seul groupe, il est facile de voir que la différence en énergie des deux fonctionnements, brutal ou graduel, depuis la même cote de départ, est seulement du deuxième ordre : en effet, l'énergie produite en fonctionnement graduel de $a$ à $b$ est :

$$
\mathrm{E}_{1}=\int_{a}^{b} n \mathrm{~N}(q, \mathrm{H}) d t
$$

or :

$$
n q=\mathrm{Q}(t)=-\mathrm{S} \frac{d z}{d t}
$$

et en chaque point :

$$
\mathbf{N}=q \frac{\partial \mathbf{N}}{\partial q}
$$

d'où :

$$
\mathrm{E}_{1}=-\int_{a}^{b} \mathrm{~S} \frac{\partial \mathrm{N}}{\partial q} d z
$$

formule rigoureuse, commode d'ailleurs pour les calculs numériques.

Au premier ordre près, la variation d'énergie sur une extrémale dans un vidage au simple effet quand on change le point de départ $z, t$ de $\delta z$ et de $\delta t$ est de :

$$
\delta \mathrm{E}=n_{\mathrm{o}}\left(\mathrm{N}-q \frac{\partial \mathrm{N}}{\partial q}\right) \delta t-\mathrm{S} \frac{\partial \mathrm{N}}{\partial q} \delta z,
$$

(Ies conditions d'arrivée restant toujours :

$$
\left.\mathbf{N}=\frac{\partial \mathbf{N}}{\partial q}=\mathbf{0}\right) \text {. }
$$

Pour chaque point de départ, on a :

$$
\mathrm{N}-q \frac{\partial \mathrm{N}}{\partial q}=\mathbf{0}
$$

Par suite, au premier ordre près, on retrouve pour l'énergie $\mathrm{E}_{2}$ consommée par abaissement de la cote de départ de $z_{a}$ à $z_{b}$ :

$$
-\int_{a}^{b} \mathrm{~S} \frac{\partial \mathrm{N}}{\partial q} d z
$$

$\mathrm{La}$ différence $\mathrm{E}_{2}-\mathrm{E}_{1}$ est donc du second ordre.

Un calcul numérique relatif à la Rance nous a montré que pour une marée moyenne (coefficient 70) la perte d'énergie est certainement inférieure $\dot{a}$ trois pour mille, elle est donc pour le moment négligeable et nous n'avons pas cherché à la calculer plus exactement.

\section{1. - ARRET EN SIMPLE EFFET}

Au vidage, par exemple, il s'agit de vider le moins possible l'amont du barrage, donc de s'arrêter le plus haut possible pour diminuer au maximum, après le remplissage sans énergie, la perte de niveau. L'arrêt brutal obtenu par le passage à zéro de tous les groupes est impossible par suite de la variation limite. L'arrêt graduel optimum aura lieu groupe par groupe.

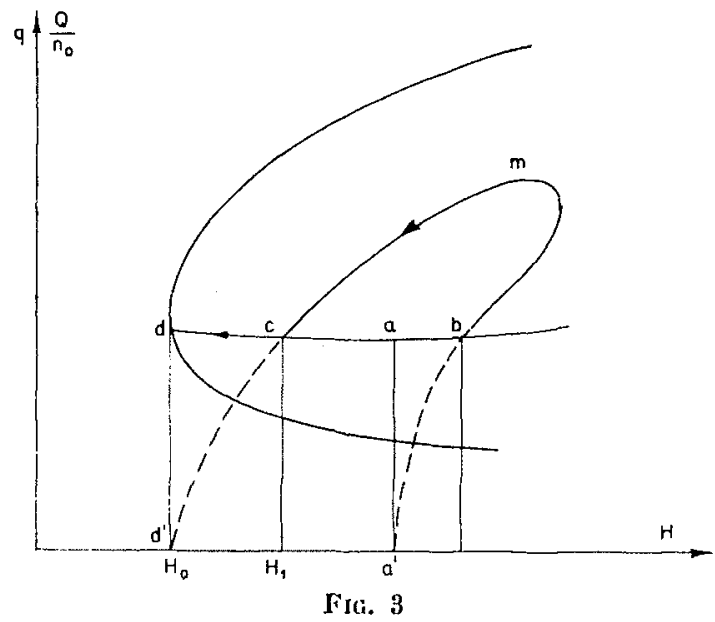

On aura $Q(t)=n q$, ici $(d Q / d t)<0$, et il s'agit de rendre maximum :

$$
E=\int n N(q, H) d t
$$

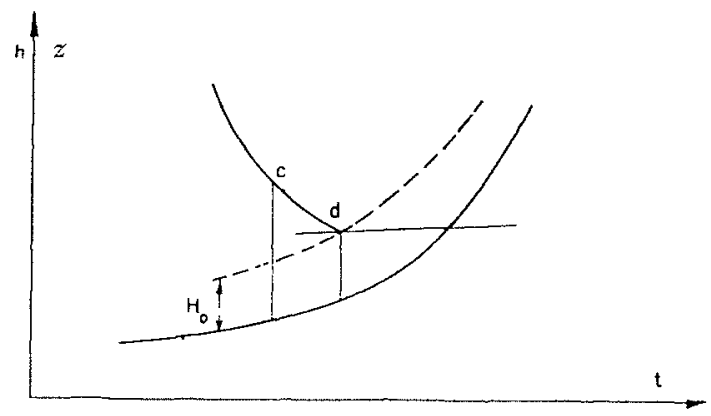

Fig. 4

Le problème est donc identique à celụi du démarrage, le point représentatif d'un groupe tournant sera situé sur la courbe de rendement optimum $\partial r_{1} \partial q=0$, le dernier groupe s'arrêtant au point classique $d$, mais la courbe $z(t)$ ayant en ce point une tangente horizontale. Par contre, 
le premier est arrêté en $c$ sous une hauteur de chute $H_{1}$ supérieure à la chute minimum, comme au démarrage le dernier groupe avait été mis en route sous une hauteur dans l'estuaire inférieure à celle $d u$ début du démarrage. Le reste de l'extrémale $b$ m $c$ est sans changement. Le calcul numérique effectué pour une marée 70 montre que la perte est certainement inférieure à deux pour mille, elle est donc négligeagle. Il est d'ailleurs possible qu'une combinaison convenable des mouvements des pales et du distributeur permette de continuer la production de tous les groupes jusqu'au point $d$ de chute minimum turbinable et à ce moment, sans perdre le synchronisme, et à puissance nulle, ou négative, mais faible en valeur absolue, de ramener le débit global progressivement à zéro. Mais tout ceci joue sur $0,1 \%$ de la production et ne trouvera son utilisation pratique que dans le simple effet. Il nous était cependant nécessaire de connaître tous ces ordres de grandeur.
Sur un graphique en $q, \mathrm{H}$, le cycle à simple effet est donc représenté, pour le groupe qui serait lancé le premier et arrêté le dernier, par la suite continue des courbes :

\section{$a b$ démarrage graduel de l'usine \\ bmc extrémale \\ $c d$ arrêt graduel de l'usine}

Notons que rien n'oblige à arrêter le dernier le groupe qui aura été lancé le premier (une hypothèse simple d'exploitation consisterait plutôt à conserver le même ordre pour le démarrage et pour l'arrêt des groupes). Le cycle relatif à un groupe quelconque partirait d'un point de démarrage situé sur $a b$ pour aboutir à un point d'arrêt sur $c d$.

Sur un graphique $\mathrm{Q} / n_{0}, \mathrm{H}$ le cycle de l'usine globale est représenté par les courbes $a^{\prime} b$ et $c d^{\prime}$ en lieux et places des courbes $a b$ et $c d$.

\section{III. - VANNAGE EN DOUBLE EFFET}

Nous voulons, par exemple au remplissage, à partir d'un point donné, gagner le plus vite possible la cote la plus haute, et cependant ne pas dépasser une loi de variation des débits $\mathbf{Q}(t)$ définie à l'avance. Nous respecterons donc cette loi. Le problème d'exploitation qui se pose est relatif à l'arrêt, progressif ou non, des groupes.

On aura ici, d'une manière générale :

$$
\mathrm{Q}(\boldsymbol{t})=n q+\left(n_{0}-n\right) \cdot q_{2}(\mathrm{H})+\mathrm{V}(\mathrm{H}),
$$

$n$ étant le nombre de groupes en fonctionnement, couplés au réseau, turbinant chacun le débit $q$,

$n_{0}$ le nombre total des groupes,

$q_{2}(\mathrm{H})$ désignant le débit en pertuis de chacun des groupes ne produisant plus d'énergie et $\mathrm{V}(\mathrm{H})$ le débit de l'ensemble des pertuis proprement dits dans leur état à l'instant $t$ considéré.

D'autre part, la puissance produite est donnée par la relation $(d \mathrm{E} / d t)=n \mathrm{~N}(q, \mathrm{H})$.

a) La loi $Q(t)$ étant fixée, une exploitation plausible est obtenue en annulant le vannage proprement dit $\mathrm{V}(\mathbf{H})$ tant qu'il y a encore des groupes en marche.

L'équation du débit global est alors :

$$
\mathrm{Q}(t)=n q+\left(n_{0}-n\right) q_{2}(\mathrm{H})
$$

d'où :

$$
n=\frac{n_{0} q_{2}-Q(t)}{q_{2}-q}
$$

$H$ étant fixée au temps $t$, l'optimum s'obtiendra, $q$ étant l'inconnue et $n$ la variable, par l'équation :

$$
\frac{\partial(n N)}{\partial q}=0 ; \frac{1}{n} \frac{\partial n}{\partial q}+\frac{1}{N} \frac{\partial N}{\partial q}=0
$$

Or :

$$
\frac{1}{n} \frac{\partial n}{\partial q}=\frac{1}{q_{2}-q},
$$

d'où l'équation :

ou :

$$
\mathrm{N}-\left(q-q_{\mathrm{s}}\right) \frac{\partial \mathrm{N}}{\partial q}=0
$$

$$
\mathrm{N}-q \frac{\partial \mathrm{N}}{\partial q}+q_{2} \frac{\partial \mathrm{N}}{\partial q}=0
$$

définissant $q$ en fonction de $\mathrm{H}$.

Cette équation, puisque $q_{2}(\mathrm{H})$ est connu, est identique à la condition de transversalité à la direction $(\delta t, \delta z)$ :

$$
n\left(\mathrm{~N}-q \frac{\partial \mathrm{N}}{\partial \eta}\right) \delta t-\mathrm{S} \frac{\partial \mathrm{N}}{\partial \eta} \delta z=0
$$

sous réserve que $S(\hat{o} z / \hat{o} t)=--n_{0} q_{2}$; la transversale à laquelle on ahoutit représente donc le 
fonctionnement en pertuis de la totalité des groupes.

Le point de fonctionnement commun des $n$ groupes est donc situé dans la représentation $(q, \mathrm{H})$ sur la courbe définie par cette équation (5) ne dépendant que de la colline et indépendante de $Q(t), z(t), \mathrm{S}(z), h(t)$ ou des conditions initiales. Cette courbe sera dite «image » de la courbe de vannage obtenue lorsque l'ensemble des groupes fonctionne en pertuis. Cette courbe conduit à faire fonctionner la turbine au-delà du point donnant le maximum de puissance (voir figure 41 de notre article S.F.E.). Cette courbe a d'ailleurs dans nos travaux été plusieurs fois envisagée comme le lieu des divers points d'arrêt, par exemple au paragraphe XXII de notre article S.F.E. (équation 60 , fig. 41) en fonctionnement classique brutal à double effet. L'introduction du changement graduel fait déplacer le point sur cette courbe, comme tout à l'heure pour le démarrage il le faisait se déplacer sur la courbe $(\partial \eta / \partial q)=0$. Les calculs précédents sont donc adaptables sans difficulté à ce résultat remarquablement simple.

La valeur $n$ se déduira de (4), la représentation graphique étant immédiate. Pour une valeur donnée de $\mathrm{H}$, soit $m$ sur la courbe d'équation (5),

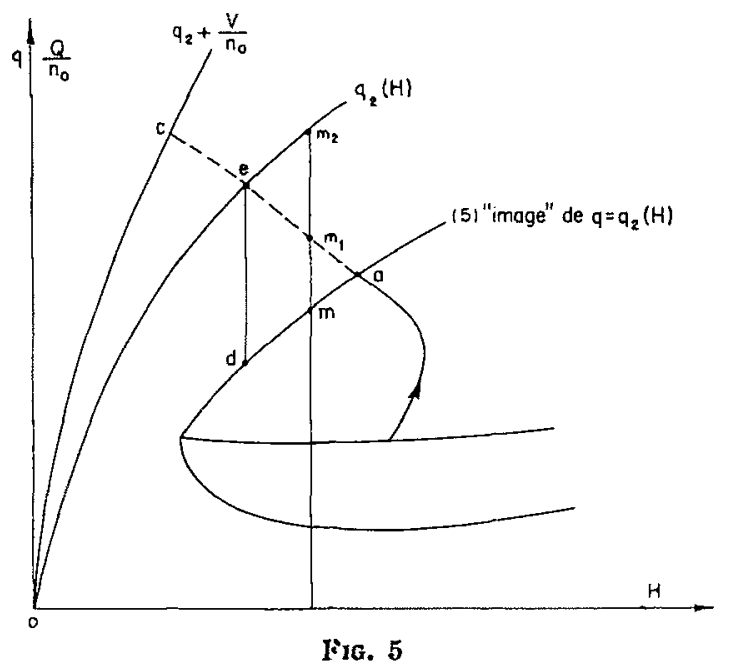

le point représentatif des groupes en marche ( $a$ correspond à $n=n_{0}, d$ à $n=0$ ); $m_{1}$ le point représentatif de la colline globale $\mathrm{Q}(\mathrm{H}) / n_{0} ; m_{2}$ le point représentatif des groupes fonctionnant en pertuis.

On peut écrire :

$$
\frac{n}{n_{0}}=\frac{m_{1} m_{2}}{m m_{2}}
$$

b) En fait, ce fonctionnement doit être complété par le retour à zéro du débit au moment où la chute s'annule. Si par exemple il a été possible de suivre une loi simple telle que $(d|\mathrm{Q}| / d t)=+3 \mathrm{~m}^{3} / \mathrm{s}^{2}$ du point $a$ au point $c$, dans la colline $\left[\left(\mathrm{Q} / n_{0}\right), \mathrm{H}\right]$, il faut revenir ensuite au débit nul par une loi $(d|\mathrm{Q}| / d t)=-3 \mathrm{~m}^{3} / \mathrm{s}^{2}$. Ces deux lois donnent deux courbes qui seront dans la représentation $(z, t)$, tangentes au point représentatif de $c$, ce qui les définit :

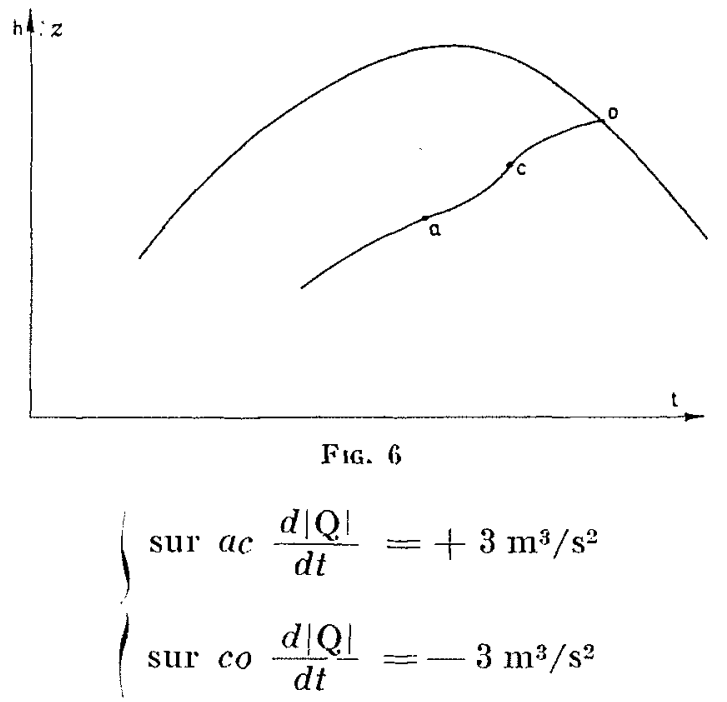

c) D'autre part, notre article S.F.E. dans certaines conditions montre que la fin d'une extrémale doit avoir lieu sur la courbe de puissance maximum $\left(\partial r_{1} / \partial q\right)=0$ (image d'un vannage infini). N'ayant pas encore la possibilité de choisir avec rigueur en utilisant les théories générales, difficiles à manier, du calcul des variations, nous avons fait faire les calculs numériques dans quatre hypothèses précises.

L'énoncé même de ces quatre hypothèses montre Ia complexité du problème dont cependant nous commençons à entrevoir la solution (voir fig. 7). Dans tous les cas, nous partons du même point $\left(z, q, \mathrm{H}_{0}, t\right)$ sur la courbe $\left(\partial \mathrm{N}^{\prime} / \partial q\right)=0$ (point $a$ ) et les calculs sont faits pour la phase de remplissage d'un cycle à double effet lors d'une marée de coefficient 70 , sur le projet 1955 de l'usine Rance (38 groupes axiaux de $7 \mathrm{MW}$ et 10 pertuis capables au total de $4.200 \mathrm{~m}^{3} / \mathrm{s}$ sous un mètre de chute).

Hypothèse A : Les 38 groupes turbinent tous ensemble suivant la loi $(\partial \mathrm{N} / \partial q)=0$ à partir $\mathrm{du}$ point $a$ vers $a^{\prime} a^{\prime \prime}$, les vannes sont ouvertes de manière à respecter la loi limite imposée au débit total de remplissage de l'estuaire $(d|\mathbf{Q}| / d t)$ $+3 \mathrm{~m}^{3} / \mathrm{s}^{2}$. Mais comme il arrive un point $a^{\prime}$ où il n'y a plus assez de vannes pour suivre la loi de $a^{\prime}$ à $a^{\prime \prime}$, on réduit le nombre de groupes en turbinage pour assurer la loi de $(d|\mathrm{Q}| / d t)$ $=+3 \mathrm{~m}^{3} / \mathrm{s}^{2}$ jusqu'en $c$, puis celle de $\left(d \mid \mathrm{Q}^{\prime} / d t\right)$ $=-3 \mathrm{~m}^{3} / \mathrm{s}^{2}$ depuis $c$ jusqu'en $c^{\prime}$. Dix groupes de cette façon sont arrêtés et fonctionnent en pertuis $\left(186 \mathrm{~m}^{3} / \mathrm{s}\right.$ sous un mètre de chute). En- 
suite, de $\alpha^{\prime \prime}$ à $b\left(^{*}\right)$, à nouveau 38 groupes en turbine, les vannes existantes étant surabondantes pour assurer le débit nécessaire pour la loi $(d|\mathrm{Q}| / d t)=-3 \mathrm{~m}^{3} / \mathrm{s}^{2}$. L'arrêt est progressif comme en simple effet et à l'arrivée à $b$ il n'y a plus de groupe en turbinage.

Rósulrat. - Depuis a il est produit poux un remplissage $78.000 \mathrm{kWh}$ et la cote atteinte à l'égalisation des niveaux mer et estuaire est $9,86 \mathrm{~m}$.
Loujours evidemment $9,86 \mathrm{~m}$ (la loi de variation du global n'ayant pas été modifiée).

L'hypothèse $B$ est donc moins favorable que l'hypothèse $A$.

Hrpotuése C: Les 33 groupes suivent l'extrémale de $\ell$ à $e$ sans vamnage supplémentaire, puis de $e$ à $d^{\prime}$ les groupes s'arrêtent progressivement et passent en pertuis jusqu'à ce que tous les groupes soient arrêtés en $d d^{\prime}$; à ce moment,

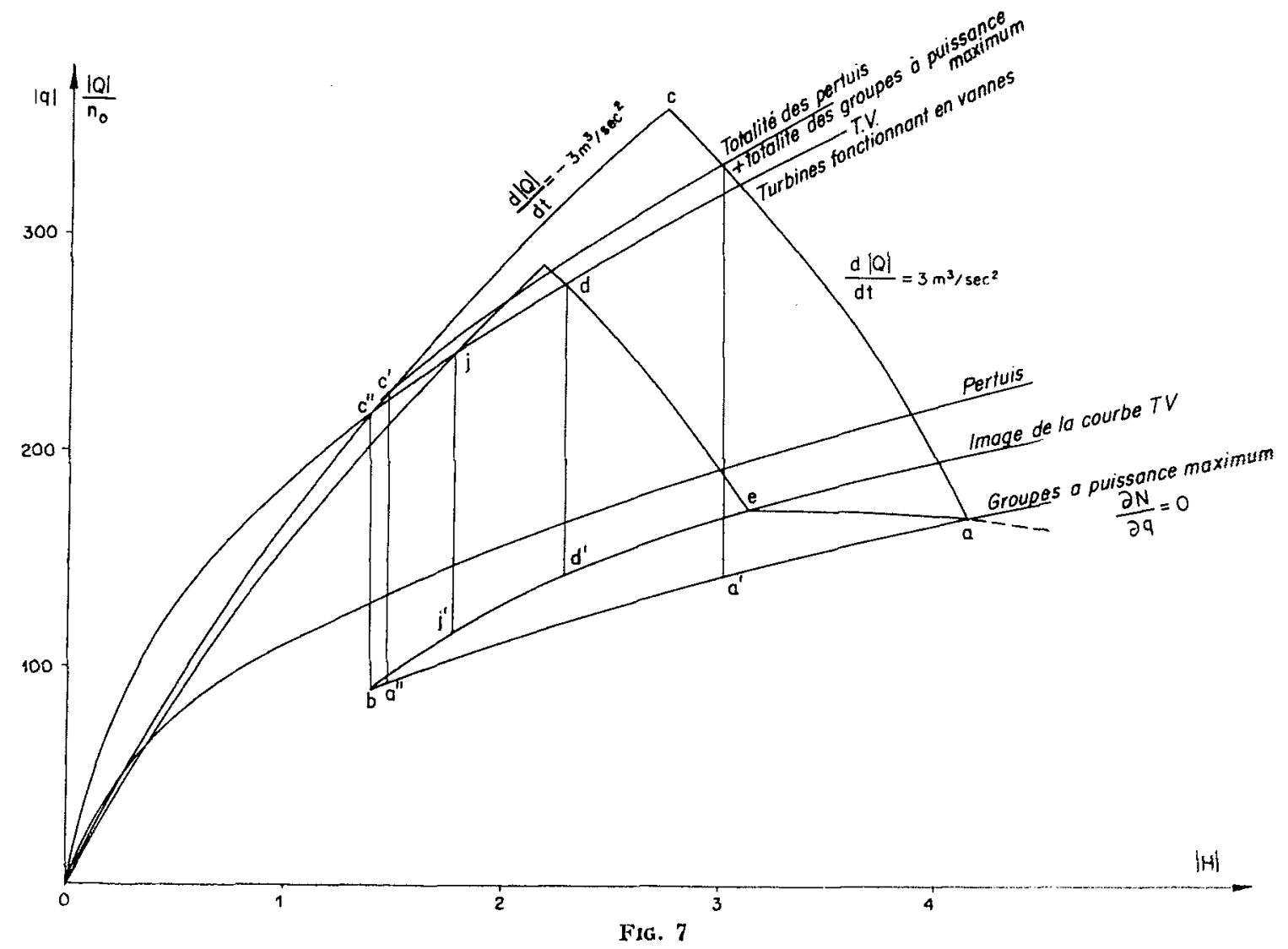

Hypothèse B : La loi du débit total n'est pas modifiée $(d \mid \mathrm{Q} ! / d t)=+3 \mathrm{~m}^{3} / \mathrm{s}^{2}$ depuis a jusqu'à $c,(d|\mathrm{Q}| / d t)=-3 \mathrm{~m}^{3} / \mathrm{s}^{2}$ de $c$ à $\mathrm{O}$. Mais les groupes tous ensemble suivent la loi $q(\mathrm{H})$ extrémale de $a$ à $e$ où on rencontre la courbe image des turbines en vannes (calcul ci-dessus), les vannes proprement dites laisant la différence, et depuis $e$ jusqu'à $b$ les 38 turbines suivent cette courbe image qui les amène à une puissance nulle en $\mathrm{O}$.

RÉsultat. -- Depuis $a$, il est produit pour un remplissage $75.000 \mathrm{kWh}$ et la cote atteinte est

(*) En résumé, le point $(|q|,|H|)$ a décrit le parcours $a a^{\prime} a^{\prime \prime} \mathrm{G}$ pendant que le point $\left[\left(|\mathrm{Q}| / n_{0}\right),|\mathrm{H}|\right]$ parcourait $a c c^{\prime} c^{\prime \prime}$. les vannes pures interviennent pour un léger parcours $d j$ et deviennent ensuite surabondantes. On peut dire qu'ici, au petil dépassement près entre $d$ et $j$, les vannes pures sont inutiles.

RÉsultat. - Depuis a il esl produit 90.000 kWh, mais le niveau atteint est ici seulement $9,58 \mathrm{ml}$. Or, les $28 \mathrm{~cm}$ de différence coutteront lors du mouvement suivant (vidage) une perle de production de $58.000 \mathrm{kWh}$, le chiffre permettant de comparer avec l'hypothèse $A$ est donc seulement de $32.000 \mathrm{kWh}$.

Hypothèse D : Le débil total suivla la même variation que dans $C$, et la cote finale restera $9,58 \mathrm{~m}$, mais le vannage se fera par des vannes 
et non par des groupes déconnectés du réseau à partir de $e$ et les 38 groupes s'arrêteront progressivement sur la courbe image de facon $\dot{a}$ ce que tous soient arrêtés en $b$.
RÉsultat. - Depuis $a$, il est produit 101.000 kWh, D est donc supérieure à $\mathrm{C}$, mais la différence de niveau ramène ce chiffrre à $43.000 \mathrm{kWh}$.

\section{CONCLUSION}

L'hypothèse A est la meilleure, mais elle ne domine $B$ que de $3.000 \mathrm{kWh}$, c'est-à-dire de $1 \%$ de l'énergie obtenue pendant tout le mouvement de remplissage. Par contre, $\mathrm{C}$ et $\mathrm{D}$ s'écartent du meilleur cycle (A) de près de $10 \%$ de l'énergie de remplissage. Ces deux chiffres 1 et $10 \%$ montrent tout à la fois l'intérêt et les limitations de nos recherches théoriques.

$$
\text { *** }
$$

On nous permettra de remercier de leur aide intelligente et dévouée MM. Penel et Voyer, de la Direction des Etudes et Recherches de l'E.D.F. Non seulement les calculs numériques ont pu, grâce à eux, être menés à bien, mais les discussions avec eux, ainsi qu'avec M. Vantroys, le chef de service du S.E.U.M., ont seules permis de mettre en évidence bien des aspects délicats de cette question difficile dont nous avons ici esquissé seulement les grandes lignes d'une théorie satisfaisante.

\section{DISCUSSION}

Président: M. BarniLLoN

M. Chamayou demande s'il n'est pas possible d'utiliser I. débit fluvial de la Rance comme « nourrice».

M. Gibrat précise que ce débit est de quelques mètres cubes/seconde, alors que celui de la mer est de l'ordre de 20000 mètres cubes/seconde. Le débit fluvial représente en temps normal environ le centième du débit d'un groupe et le débit de la crue centenaire reste inférieur à ce dernier.

Le débit fluvial de la Rance est donc négligeable de toute façon.

M. le Président remercie M. Gibrat de son brillant exposé.

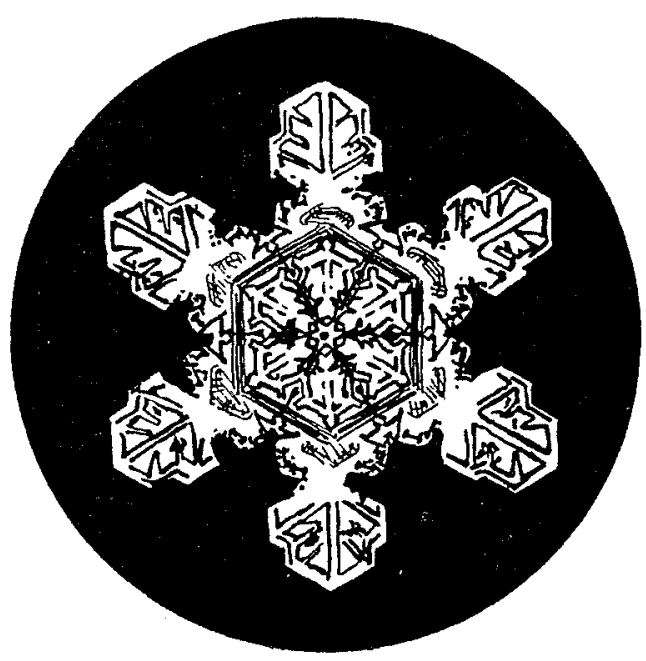

